\title{
Circadian clock gene Per2 downregulation in non-small cell lung cancer is associated with tumour progression and metastasis
}

\author{
RUN XIANG $^{1 *}$, YUE CUI $^{2 *}$, YANPING WANG $^{3}$, TIANPENG XIE $^{1}$, XIAOJUN YANG $^{1}$, \\ ZHU WANG ${ }^{3}, \mathrm{JUAN} \mathrm{LI}^{4}$ and QIANG LI ${ }^{1}$ \\ ${ }^{1}$ Department of Thoracic Surgery, Sichuan Cancer Hospital and Institute, Sichuan Cancer Center, \\ School of Medicine, University of Electronic Science and Technology of China, Chengdu, Sichuan 610041; \\ ${ }^{2}$ Department of Thoracic Surgery, First Affiliated Hospital of Kunming Medical University, Kunming, \\ Yunnan 650000; ${ }^{3}$ Laboratory of Molecular Diagnosis of Cancer, West China Hospital, Sichuan University; \\ ${ }^{4}$ Department of Chemotherapy, Sichuan Cancer Hospital and Institute, Sichuan Cancer Center, School of Medicine, \\ University of Electronic Science and Technology of China, Chengdu, Sichuan 610041, P.R. China
}

Received March 25, 2018; Accepted September 3, 2018

DOI: $10.3892 / o r .2018 .6704$

\begin{abstract}
Period2 (Per2) is a key circadian clock gene, and its deregulation contributes to tumour development, including breast cancer. However, the biological function and clinicopathological significance of Per2 in non-small cell lung cancer (NSCLC) remain unclear. The present study aimed to explore the role of Per2 and its relative clinical significance in NSCLC. To analyse Per2 expression in NSCLC specimens, reverse transcription-quantitative polymerase chain reaction was performed, and the results indicated that Per2 expression was markedly downregulated in $83.87 \%$ (26/31) of NSCLC samples compared with their adjacent matched tissues. Increased Per2 expression was associated with increased differentiation $(\mathrm{P}<0.01)$ and reduced lymph node metastasis $(\mathrm{P}<0.0001)$. Functional studies identified that enhancing Per 2 expression in A549 cells by lentivirus transduction not only significantly suppressed cell growth, migration and invasion $(\mathrm{P}<0.05)$ but also inhibited NSCLC growth and metastasis in vivo. Animal
\end{abstract}

Correspondence to: Professor Qiang Li, Department of Thoracic Surgery, Sichuan Cancer Hospital, 55 Renmin South Road, Chengdu, Sichuan 610041, P.R. China

E-mail: liqiang201320@163.com

Professor Juan Li, Department of Chemotherapy, Sichuan Cancer Hospital, 55 Renmin South Road, Chengdu, Sichuan 610041, P.R. China

E-mail: dr.lijuan@hotmail.com

${ }^{*}$ Contributed equally

Abbreviations: NSCLC, non-small-cell lung cancer; Per1, Period1; Per2, Period2; Per3, Period3; Cry1, cryptochrome1; VEGF, vascular endothelial growth factor; $\mathrm{NC}$, negative control

Key words: non-small-cell lung cancer, Period2, metastasis, proliferation studies and histopathological analysis identified that Per2 expression in A549 cells not only markedly increased expression of tumour anti-oncogenes Bax, P53 and P21 but also inhibited expression of pro-oncogenes vascular endothelial growth factor, CD44 and c-Myc. These results indicate that the loss of Per2 is one of the factors underlying tumourigenesis in NSCLC, and it may function as a novel molecular target for NSCLC.

\section{Introduction}

Lung cancer is the most common cancer type, which is responsible for around 1.4 million deaths worldwide each year (1). NSCLC represents more than $80 \%$ of lung cancer cases (2). Although there have been advances in surgical techniques, chemotherapy and radiotherapy for treating lung cancer, the overall survival of patients remains low $(3,4)$. Therefore, it is important to investigate a more complete system for prognostic evaluation. It is necessary to identify a more useful prognostic and predictive markers for early molecular diagnosis or potential targets for more precise risk analysis and development of new therapies.

The daily cycle of light and dark is a driving force for various living organisms. Therefore, circadian rhythms are basic regulators of an organism's biological activities. Circadian rhythms are daily oscillations that are regulated by endogenous clock genes. They make it easier for organisms to adapt to daily environmental changes, including temperature, pressure and light. They are also able to synchronize multiple molecular, biochemical, physiological and behavioural processes $(5,6)$. Common core clock genes include CLOCK, BMAL1, Period1 (Per1), Period2 (Per2), Period3 (Per3), cryptochrome1 (Cry1), cryptochrome2, casein kinasel epsilon and timeless (7). A number of epidemiological studies have suggested that disruption of the normal circadian rhythm may increase the risk of developing various types of cancer, including breast (7), prostate (8), colorectal, liver and endometrial cancers (9-11). Among all known clock genes, only the members of the Per 
subfamily function as tumour suppressors in mice (8). The neoplastic growth of cancer cells may be restrained by overexpression of Per1 or Per2, and their apoptotic rate may also be increased (8). Importantly, the involvement of Per1 and Per2 in ataxia telangiectasia mutated checkpoint kinase DNA damage response pathways implicates the circadian system in tumour suppression (9).

It has been reported that Per1 is downregulated in NSCLC cell lines and inhibits cancer cell proliferation (7). In addition, Couto et al (10) reported that Per3 was downregulated in NSCLC, and polymorphisms in Per3 genes may be a factor underlying NSCLC in Brazilian patients. According to these reports, the members of the Per subfamily appear to be responsible for tumour progression in NSCLC. However, the biological function and clinicopathological significance of Per2 in NSCLC remains unclear. In the present study, the role of Per2 and its relative clinical significance in NSCLC were explored. It was identified that loss of Per2 was associated with NSCLC progression, and recovery of Per2 expression could suppress A549 cell growth and migration in vitro and in vivo, suggesting that Per2 may serve as a potential molecular target for NSCLC.

\section{Materials and methods}

Clinical samples. A total of 31 pairs of NSCLC and matched para-carcinoma tissues were collected from the Department of Thoracic Surgery, Sichuan Cancer Hospital and Institute (Sichuan, China) between March 2015 and May 2016. All patients were between 30 and 78 years old and among them 20 were male and 31 were female. All patients provided written informed consent, and the study was approved by the Ethical Committee of Sichuan Cancer Hospital \& Institute. Lung cancer tissues and matched para-carcinoma tissues were immediately frozen in liquid nitrogen following surgical resection and then stored at $-80^{\circ} \mathrm{C}$ until use. The differentiation and metastasis states were identified by experienced pathologists.

Cell culture and lentivirus transduction. A549 cells were purchased from the Institute of Biochemistry and Cell Biology of Chinese Academy of Science (Shanghai, China) and cultured in RPMI-1640 (Hyclone; GE Healthcare Life Sciences, Logan, UT, USA) with $15 \%$ foetal bovine serum (FBS; Gibco; Thermo Fisher Scientific, Inc., Waltham, MA, USA) at $37^{\circ} \mathrm{C}$ in a humidified incubator containing $5 \% \mathrm{CO}_{2}$. Overexpression lentivirus of Per2 was purchased from Genechem (Shanghai GeneChem Co., Ltd., Shanghai, China). A549 cells were infected with virus at a multiplicity of infection of 60, and the expression rate of green fluorescence protein was observed with a fluorescence microscope (IX70; Olympus Corporation, Tokyo, Japan) on day 3. Finally, the infected efficiency of A549 following $72 \mathrm{~h}$ of lentiviral transduction was confirmed by reverse transcription-quantitative polymerase chain reaction (RT-qPCR) and western blot analysis.

Wound healing assay. A549 cells at a density of $3 \times 10^{5}$ cells/well were seeded in 6-well culture plates and cultured overnight at $37^{\circ} \mathrm{C}$. Then, a sterile, plastic $200-\mu 1$ micropipette tip was used to make a straight scratch on the confluent cell monolayer. The well was washed with medium to remove detached cells, then cells were cultured for a further $48 \mathrm{~h}$ at $37^{\circ} \mathrm{C}$. Finally, images were obtained under a light microscope and the width of the wound was measured with the microscope and ImageJ software v1.37 (National Institutes of Health, Bethesda, MD USA).

Cell proliferation and colony formation assays. A549 cells with or without lentiviral infection were seeded at a density of $1.5 \times 10^{3}$ cells/per well into 96 -well plates. The cell growth of A549 cell lines was determined by Cell Counting Kit-8 (CCK-8) assay (Dojindo Molecular Technologies, Inc., Kumamoto, Japan) once a day for 1 week. Assays of each group were performed in quintuplicate wells.

For colony formation assays, A549 cells with or without lentiviral transduction were seeded into 6 -well plates at a low density (200 cells/per well) and cultured for 2 weeks. Then, cells were fixed with $4 \%$ paraformaldehyde at $37^{\circ} \mathrm{C}$ for $15 \mathrm{~min}$ and stained with $1 \%$ crystal violet for $30 \mathrm{~min}$ at room temperature. Finally, the number of colonies of substantial size (>50 cells per colony) were imaged by light microscope, with three fields of view per well. The experiments were repeated in triplicate.

Cell cycle analysis by DNA content. Briefly, A549 cells with or without lentiviral transduction for $48 \mathrm{~h}$ were harvested and washed by centrifugation at $200 \mathrm{x}$ g for $5 \mathrm{~min}$ at $25^{\circ} \mathrm{C}$ in $1 \mathrm{X}$ PBS buffer. Next, cells were fixed in $70 \%$ ethanol at $4^{\circ} \mathrm{C}$ overnight and washed twice with cold $1 \mathrm{X}$ PBS buffer. Then, cells were resuspended in $100 \mu \mathrm{l}$ RNase A $(10 \mu \mathrm{g} / \mathrm{ml})$, incubated at $37^{\circ} \mathrm{C}$ for $30 \mathrm{~min}$ and immediately stained with $400 \mu \mathrm{l}$ propidium iodide $(500 \mu \mathrm{g} / \mathrm{ml}$; Nanjing KeyGen Biotech Co., Ltd., Nanjing, China) at $4^{\circ} \mathrm{C}$ for $30 \mathrm{~min}$ in the dark. Finally, data were acquired on a flow cytometer in triplicate (Coulter Epics XL; Beckman Coulter, Inc., Brea, CA, USA) and analysed by Flow Jo7.6 software (FlowJo LLC, Ashland, OR, USA).

In vitro migration and invasion assays. Migration assays and invasion assays were performed using 24-well Transwell filters with $8 \mu \mathrm{m}$ pore size polycarbonate membrane (Corning, Inc., Corning, NY, USA) and an invasion assay kit that included Matrigel (BD Biosciences, San Jose, CA, USA). Briefly, $48 \mathrm{~h}$ following transfection, $2 \times 10^{4}$ (for the migration assay) or $1 \times 10^{5}$ cells (for the invasion assay) were resuspended in $200 \mu \mathrm{l}$ serum-free RPMI-1640 and plated into the upper chamber. The bottom of the lower chambers in the 24-well plates was filled with $0.6 \mathrm{ml}$ medium containing $10 \% \mathrm{FBS}$, which was used as a chemoattractant. Following incubation at $37^{\circ} \mathrm{C}$ for $48 \mathrm{~h}$, the Transwell inserts were removed from the plate, and a cotton-tipped applicator was used to carefully remove the media and remaining cells that had not migrated or invaded from the top of the membrane without damaging it. Then, cells were fixed with $600 \mu \mathrm{l}$ of $4 \%$ paraformaldehyde, stained with $0.5 \%$ crystal violet and incubated at room temperature for $5 \mathrm{~min}$ at $37^{\circ} \mathrm{C}$. Then, the Transwell membranes were allowed to dry. The lower chamber was photographed by a light microscope and cells were counted in at least five different fields of view.

Hematoxylin and eosin $(H \& E)$ staining. Xenograft tissues were immersed in $4 \%$ paraformaldehyde at $37^{\circ} \mathrm{C}$ for $4 \mathrm{~h}$, then 
transferred to $70 \%$ ethanol. Individual lobes of xenograft tissue biopsy material were placed in processing cassettes, dehydrated through a serial alcohol gradient, and embedded in paraffin wax blocks. Prior to staining, 5- $\mu \mathrm{m}$-thick lung tissue sections were dewaxed in xylene, rehydrated through decreasing concentrations of ethanol, and washed in PBS. Tissues were then stained at room temperature with H\&E. Then, sections were dehydrated through increasing concentrations of ethanol and xylene. A light microscope was used to examine the tissues.

Immunohistochemistry (IHC) analysis. The tumor xenograft were immersed in $4 \%$ paraformaldehyde at $37^{\circ} \mathrm{C}$ for $4 \mathrm{~h}$, and $5 \mu \mathrm{m}$ thick slices were used for IHC staining by the streptavidin-biotin-peroxidase complex method. The antigen retrieval procedure was performed by heating the samples in antigen retrieval solution containing $10 \mathrm{mM}$ sodium citrate buffer with a pressure cooker at $100^{\circ} \mathrm{C}$ for $150 \mathrm{sec}$. When the slices were cooled, normal goat serum blocking solution (Beyotime Institute of Biotechnology, Shanghai, China) was used for blocking at $37^{\circ} \mathrm{C}$ for $2 \mathrm{~h}$. Rabbit anti human Per2 (1:200; ab179813; Abcam, Cambridge, MA, USA), Ki-67 (1:500; ab8191; Abcam), E-Cadherin (1:100; ab133597; Abcam) and NM23 (1:100; ab154529; Abcam) were incubated at $4^{\circ} \mathrm{C}$ overnight. Then, the Dako REAL ${ }^{\text {TM }}$ EnVision $^{\text {TM }}$ kit (K500711; Dako; Agilent Technologies, Inc., Santa Clara, CA, USA) was used to incubate the slices at $37^{\circ} \mathrm{C}$ for $30 \mathrm{~min}$. IHC staining intensity was independently scored under a light microscope by two anatomical pathologists. The staining intensity (negative $=0$, weak $=1$, moderate $=2$ or strong $=3$ ) and the proportion of positively stained cells $(<25 \%=1,25-50 \%=2$, $>50$ and $<75 \%=3, \geq 75 \%=4$ ) were scored. The immunostaining was semi-quantitatively categorized by multiplying the intensity and the quantity scores to yield a staining index (values from 0 to 12). A staining index of 3-12 was regarded as high expression, while a staining index of $0-2$ was regarded as low expression.

Animal studies. This experiment was conducted in accordance with the Care and Use of Laboratory Animals (12) and was approved by the Ethical Committee of Sichuan Cancer Hospital \& Institute. Sixteen 4-week-old male BALB/c-nude mice weighing 12-15 $\mathrm{g}$ were purchased from the Laboratory Animal Centre of Sichuan University (Chengdu, China) and reared in specific-pathogen-free conditions. The mice were randomly and equally divided into experimental and control groups, in which the experimental group mice underwent tumour cell transplantation with Per2 overexpression and the control group mice underwent tumour cell transplantation without Per2 overexpression. For the subcutaneous transplantation tumour model, $2 \times 10^{6}$ A549 cells that did or did not overexpress Per2 were resuspended in $0.1 \mathrm{ml}$ PBS and then injected subcutaneously into the backs of the mice. Tumour growth was measured with Vernier calipers over the course of 30 days following transplantation of tumour cells, and tumour volume was calculated according to the formula: Tumour volume $=0.5 \mathrm{x}$ length $\mathrm{x}$ width $^{2}$. For the lung metastasis model, $1 \times 10^{5}$ A549 cells diluted in $100 \mu 1$ PBS was intravenously injected via the mouse tail vein. The metastasis score was determined by the pathological condition of the metastatic mice following treatment for 4 weeks.
$R N A$ extraction and $R T-q P C R$. Total RNA was extracted from cells using a Total RNA kit (Omega Bio-Tek, Inc., Norcross, GA, USA) according to the manufacturer's protocol. Reverse transcription to cDNA was perfroemd using Prime Script ${ }^{\mathrm{TM}}$ RT Master mix (Takara Biotechnology Co., Ltd., Dalian, China). The cDNA was used for qPCR using the CFX96 Real-Time Quantitative PCR system (Bio-Rad Laboratories, Inc.) with SYBR ${ }^{\circledR}$ Premix ExTaq ${ }^{\mathrm{TM}}$ (Takara Biotechnology Co., Ltd.) according to the manufacturer's protocol. Relative mRNA expression levels were determined by the cycle threshold $(\mathrm{Ct})$ normalized against GAPDH using the $2^{-\Delta \Delta \mathrm{Ct}}$ formula (13). Experiments were performed in triplicate. The primers used in this study were as follows (5'-3'): Per2-forward (F), CAGGTGAAAGCCAATGAAGAG; Per2-reverse (R), GGGAGGTGAAACTGTGGAAC; Bax-F, CCCGAGAGG TCTTTTTCCGAG; Bax-R, CCAGCCCATGATGGTTCT GAT; P53-F, ACTTGTCGCTCTTGAAGCTAC; P53-R, GAT GCGGAGAATCTTTGGAACA; P21-F, CCAACAAACTTA ACGTGCCAC; P21-R, AGGCTCAACAGTAACTGCATC; Nm23-F, CATTGCGATCAAACCAGATG; Nm23-R: CAG AAGTCTCCACGGATGGT; E-Cadherin-F, CGAGAGCTA CACGTTCACGG; E-Cadherin-R, GGGTGTCGAGGGAAA AATAGG; vascular endothelial growth factor (VEGF)-F, GGAGGAGGAAGAAGAGAAGGAA; VEGF-R, GTGGAG GTAGAGCAGCAAGG; CD44-F, CAGCACCATTTCAAC CACAC; CD44-R, GCCAAACCACTGTTCCTTCT; cMyc-F, CCGAGGAGAATGTCAAGAGG; cMyc-R, ACGCACAAG AGTTCCGTAGC; GAPDH-F, GAAGGTGAAGGTCGG AGTC; and GAPDH-R, GAAGATGGTGATGGGATTTC.

Western blot analysis. Antibodies for Per2 (ab179813), p53 (\#48818), BAX (\#2774), P21 (\#2947), E-Cadherin (\#14472), NM23 (\#3338), VEGF (\#2463), CD44 (\#3570), c-Myc (\#5605) and $\beta$-actin (\#3700) were purchased from Abcam or Cell Signaling Technology, Inc. (Danvers, MA, USA) and all the antibodies were rabbit anti-human. Cells were harvested, lysed with RIPA buffer (Sigma-Aldrich; Merck KgaA, Darmstadt, Germany) supplemented with $1 \mathrm{mmol} / 1$ PMSF (Boster Biological Technology, Pleasonton, CA, USA) and then centrifuged at $14,000 \mathrm{xg}$ at $4^{\circ} \mathrm{C}$ for $10 \mathrm{~min}$. Protein concentrations of the extracts were measured using a bicinchoninic acid protein assay kit (Nanjing KeyGen Biotech Co., Ltd.). Proteins ( $20 \mu \mathrm{g}$ ) were separated through $10 \%$ SDS-PAGE gels, and then transferred to polyvinylidene difluoride membranes. Following blocking in Tris-buffered saline with Tween-20 containing 5\% non-fat milk for $60 \mathrm{~min}$, the membranes were incubated with primary antibodies (P53, Bax, c-Myc, Per2, NM23, CD44, E-cadherin and VEGF primary antibody: 1:1,000 dilution; P21 primary antibody: $1: 500$ dilution; $\beta$-actin as a loading control: 1:2,500 dilution) overnight at $4^{\circ} \mathrm{C}$. The membranes were then incubated with the secondary antibodies(mouse anti-rabbit and horseradish peroxidase-linked antibody, 1:5,000 dilution; Cell Signaling Technology, Inc.). Following incubation in enhanced chemiluminescence solution, the proteins on the membranes were detected using Bio-Rad Universal Hood III and analysed using Image $\mathrm{Lab}^{\mathrm{TM}}$ software 2.0 (Bio-Rad Laboratories, Inc.).

Statistical analysis. Data are presented as the mean \pm standard deviation, with the exception of tissue expression data, which are presented as the median \pm range. The differences 

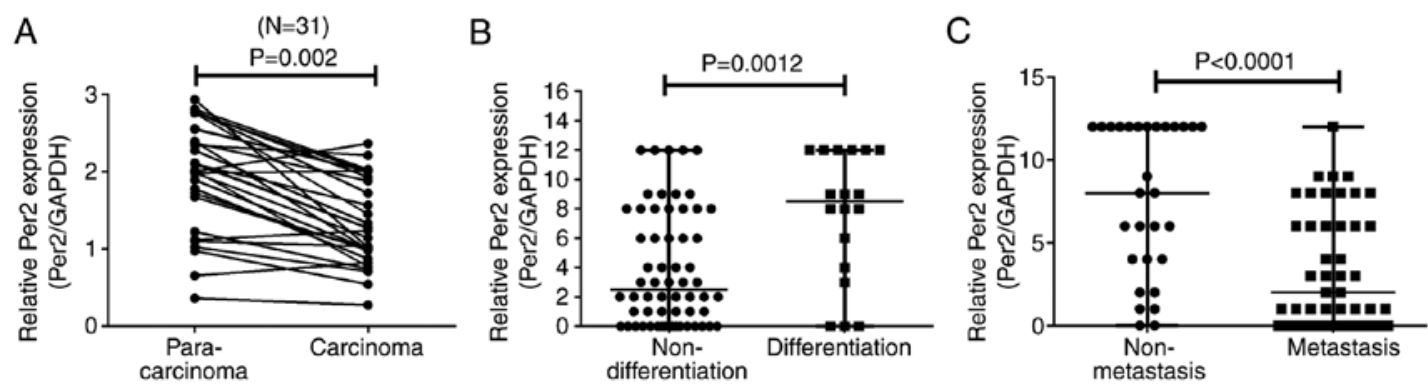

Figure 1. Per2 expression is decreased in NSCLC tissues and associated with clinicopathological characteristics. (A) Per2 expression levels in 31 paired NSCLC tissues and their matched controls were analysed by reverse transcription-quantitative polymerase chain reaction. Evaluation of clinicopathological characteristics identified that low expression of Per2 was significantly associated with (B) pathological differentiation and (C) lymph node metastasis. Data are presented as the median \pm range. Per2, Period2; NSCLC, non-small-cell lung cancer.

in Per2 expression between NSCLC tissues and matched para-carcinoma adjacent normal lung tissues were compared by a two-tailed independent samples Student's t-test. All analyses were performed using GraphPad Prism 6.0 software (GraphPad Software, Inc., La Jolla, CA, USA). P<0.05 was considered to indicate a statistically significant difference.

\section{Results}

Per2 is downregulated in NSCLC tissues and associated with clinicopathological characteristics. Per2 gene exhibits low expression in certain cancer tissues. In the present study, the expression of Per2 in lung cancer was evaluated by performing RT-qPCR in different histological subtypes of NSCLC tissue. As presented in Fig. 1A, compared with matched controls, the Per2 mRNA level was strongly downregulated in $83.87 \%$ (26 of 31) of NSCLC samples ( $\mathrm{P}<0.01$ vs. control). As indicated in Fig. $1 \mathrm{~B}$ and $\mathrm{C}$, Per2 expression was significantly associated with pathological differentiation $(\mathrm{P}<0.01)$ and lymph node metastasis $(\mathrm{P}<0.0001)$. Increased Per 2 expression indicated increased differentiation and reduced lymph node metastasis. These findings suggested that Per2 may be involved in NSCLC development.

Overexpression of Per2 decreases A549 cell growth by blocking the cell cycle at G0/G1 phase. Based on the finding that Per2 expression was decreased in NSCLC samples, a Per2-expressing lentivirus was constructed and used to infect A549 cells, in order to determine the potential role of Per2 in NSCLC. As presented in Fig. 2A, the mRNA expression of Per2 increased 11-fold and Per2 protein expression also increased in A549 cells following infection with Per2-expressing lentivirus. Next, a CCK-8 assay, colony formation assay and cell cycle analysis were performed to detect the effect of overexpression of Per2 on A549 cell growth. The results of the CCK-8 and colony formation assays demonstrated that overexpression of Per2 suppressed A549 cell proliferation and colony formation compared with the control group $(\mathrm{P}<0.05$; Fig. 2B and C). Furthermore, flow cytometry was performed to analyse the influence of Per2 on the cell cycle and it was identified that the percentage of A549 cells that remained in G1 phase was significantly increased in the Per2 overexpression group compared with the control group $(76.67 \pm 10.22$ vs. $66.80 \pm 11.38 \%$; $\mathrm{P}<0.01$ ), while fewer cells stayed in $\mathrm{S}$ phase compared with the control group $(6.01 \pm 2.74$ vs. $10.03 \pm 3.01 \%)$ after $48 \mathrm{~h}$ of culture (Fig. 2D). These results indicated that increased Per2 expression in A459 cells inhibits tumour cell proliferation by inducing cell cycle arrest at G0/G1 phase.

Overexpression of Per2 suppresses A549 cell migration and invasion ability. Metastasis begins when the tumour invades into the vascular stroma and migrates to the blood (14). Therefore, the influence of Per2 on tumour cell migration and invasion ability was evaluated with in vitro Transwell assays. Overexpression of Per2 significantly inhibited the number of migrating and invading A549 cells compared with the negative control group (migration: $130.80 \pm 9.987$ vs. $20.20 \pm 4.164$; invasion: $134.20 \pm 7.165$ vs. $56.80 \pm 6.763$; both $\mathrm{P}<0.0001$; Fig. $3 \mathrm{~A}$ ). Similarly, results of the wound healing assay revealed that wound gap closure and migration was significantly decreased for A549 cells overexpressing Per2 ( $\mathrm{P}<0.01$; Fig. 3B). These results demonstrated that Per2 may act as an anti-oncogene in suppressing A549 cell growth and metastasis.

Overexpression of Per2 decreases apoptosis-and metastasis-associated gene and protein expression in A549 cells. To further elucidate the molecular mechanisms underlying Per2 effects on cell growth and metastasis, apoptosis-associated genes Bax, P53, P21 and NM23, metastasis-associated genes E-Cadherin, VEGF and CD44, and c-Myc oncogene were evaluated. RT-qPCR and western blotting results are presented in Fig. 4. Overexpression of Per2 markedly increased the expression of Bax, P53, P21 and NM23 and suppressed VEGF, CD44 and c-Myc expression. These findings indicated that the antitumour effect of Per2 is associated with these apoptosisand metastasis-associated genes.

Overexpression of Per2 reduces tumour formation of A549 cells in a nude mouse xenograft model. To evaluate whether Per2 exhibited antitumour effects in vivo, A549 cells that were or were not transduced with Per2-overexpressing lentivirus were subcutaneously implanted into the backs of BALB/c-nude mice. Consistent with the current in vitro results, Per2-lentivirus A549 NSCLC cells exhibited delayed tumour formation compared with negative controls $(\mathrm{P}<0.05$; Fig. 5A). At 30 days after implanting tumour cells, the tumour weight of the Per2-overexpressing group was $0.328 \pm 0.171 \mathrm{~g}$, while that of the negative control group was $0.785 \pm 0.249 \mathrm{~g}$; therefore, 

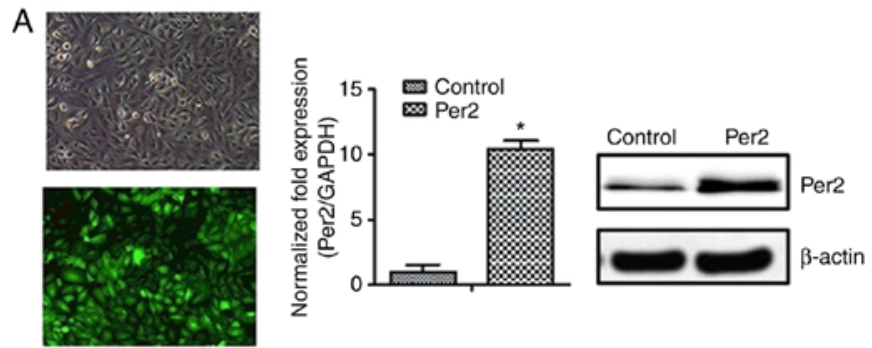

$\mathrm{C}$

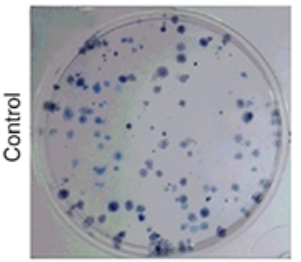

D

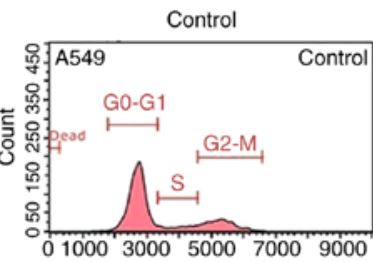

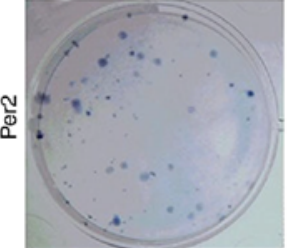

Pere

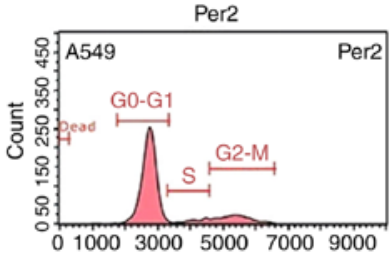

B

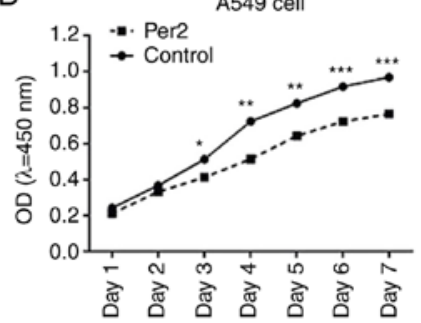

Figure 2. Overexpression of Per2 in A549 cells decreases tumour cell growth by blocking the cell cycle at G0/G1 phase. (A) Overexpression of Per2 in A549 cells was performed by lentivirus infection. Magnification, x40. Per2 overexpression in A549 cells was verified by reverse transcription-quantitative polymerase chain reaction and western blotting. (B) The effect of Per2 overexpression on A549 cell proliferation was evaluated using Cell Counting Kit-8 assay at the indicated time points. (C) The effect of Per2 on colony formation of A549 cells was evaluated at 2 weeks. (D) The cell cycle distribution of A549 cells transduced with Per2-overexpressing lentivirus or negative control for $48 \mathrm{~h}$ was analysed by flow cytometry. Similar results were obtained in three experiments. ${ }^{*} \mathrm{P}<0.05,{ }^{* *} \mathrm{P}<0.01,{ }^{* * *} \mathrm{P}<0.001$. Per 2 , cells transfected with lentiviral vector overexpressing Period 2 .

A

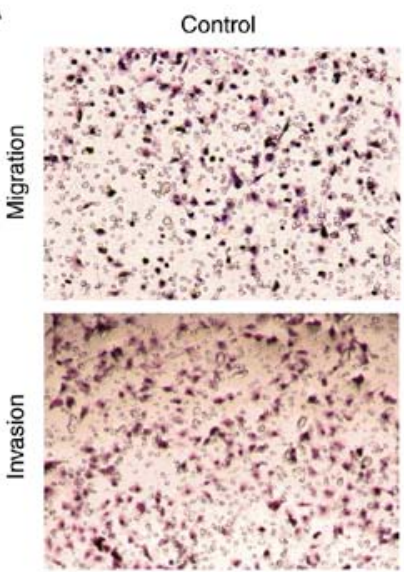

Per2

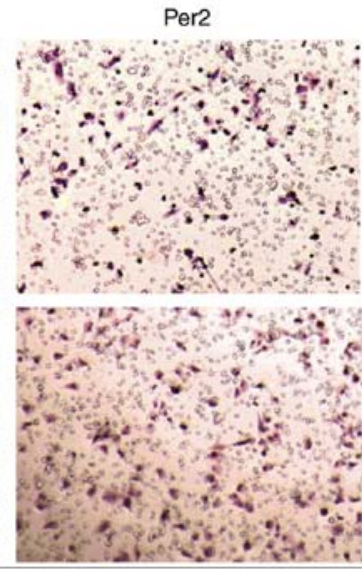

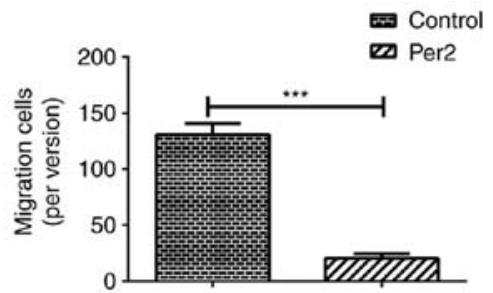

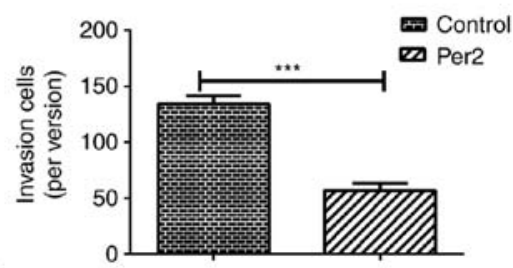

B
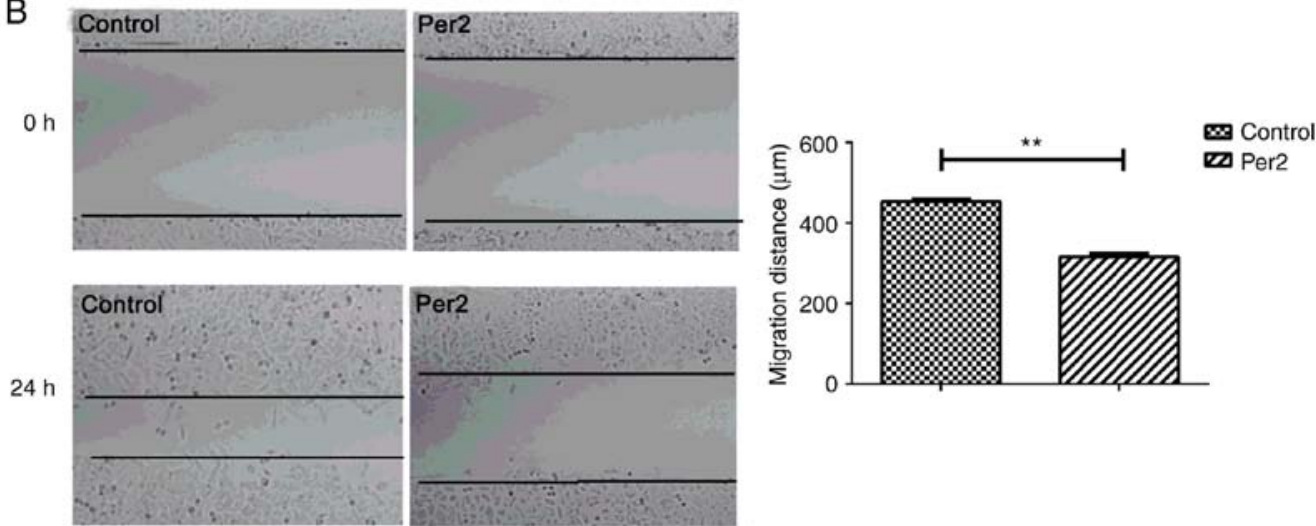

Figure 3. Overexpression of Per2 suppresses A549 cell migration and invasion. (A) Transwell assays were used to analyse the effect of Per2 on A549 cell migration and invasion ability (magnification, $\mathrm{x} 40$ ). (B) Wound healing assay was performed to assess the effect of Per2 on A549 cell migration capacity. Data are presented as the mean \pm standard deviation from three independent experiments. ${ }^{* *} \mathrm{P}<0.01,{ }^{* * * *} \mathrm{P}<0.001$. Per2, cells transfected with lentiviral vector overexpressing Period2. 


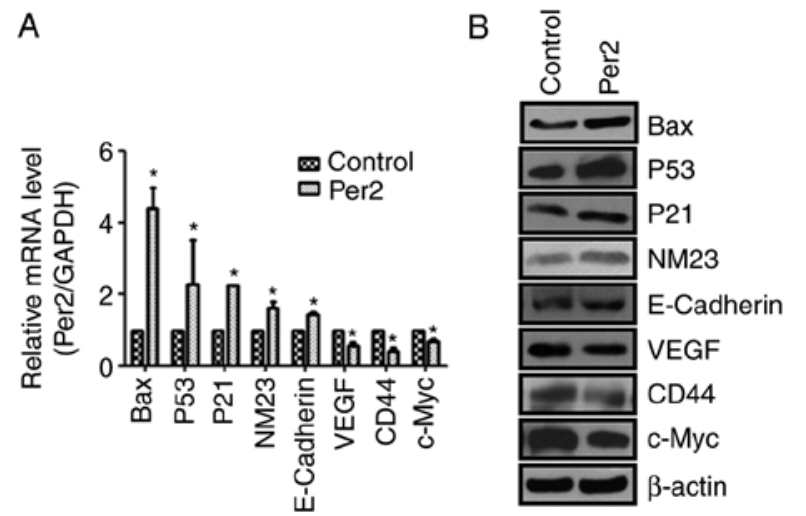

Figure 4. Overexpression of Per2 decrease apoptosis- and metastasis-associated gene and protein expression in A549 cells. Changes in (A) gene and (B) protein expression of Bax, P53, P21, NM23, E-Cadherin, VEGF, CD44 and c-Myc in A549 cells following transduction of Per2-overexpressing lentivirus or negative control for $48 \mathrm{~h}$ were detected by reverse transcription-quantitative polymerase chain reaction and western blot analysis, respectively. Per2, cells transfected with lentiviral vector overexpressing Period2; VEGF, vascular endothelial growth factor. ${ }^{*} \mathrm{P}<0.05$.

the tumour growth inhibition rate of the Per2-overexpressing group was $63.56 \%(\mathrm{P}<0.05$; Fig. 5B). Furthermore, the number of lung metastasis tumours in the Per2-overexpressing group was significantly decreased when compared with those of the control group, and the H\&E staining identified less metastasis in the Per2-overexpressing group (Fig. 5C and D). In addition, IHC analysis was performed on animal tumour samples. As indicated in Fig. 5E, the score of Per2 expression in the Per2-overexpression group $(6.38 \pm 2.504)$ was increased compared with the control group (3.12 \pm 2.232$)$. For E-cadherin, NM23 and Ki-67 protein expression, IHC indicated that overexpression of Per2 could increase expression of the tumour growth suppressor E-cadherin and tumour metastasis inhibitor NM23, and lower the expression of Ki-67. Collectively, these results suggested that overexpression of Per2 reduces the capacity of A549 cells to form tumours in vivo.

\section{Discussion}

Core circadian genes play an important role in tissue homeostasis and tumourigenesis. Multiple cancer types are commonly caused by the disruption of circadian rhythms (15). Thus, improved understanding of the underlying biological role of circadian rhythms in NSCLC would help to elucidate the occurrence and development of lung cancer and bring about new ideas for NSCLC diagnosis and therapy. In the current study, it was identified that compared with adjacent non-cancerous tissues, Per2 expression was strongly downregulated in NSCLC samples and reduced expression was associated with pathological non-differentiation and lymph node metastasis. Functional studies identified that enhanced Per2 expression in A549 non-small cell lung cancer cells significantly suppressed cell proliferation, migration and invasion,7 and inhibited NSCLC growth and metastasis in vivo. Therefore, Per2 may be a novel molecular target for NSCLC.

Numerous studies have confirmed that circadian rhythm dysfunction is associated with the pathogenesis of cancer. Disruption of circadian rhythms may promote tumour progression, and restored circadian rhythms may potentially have a positive effect on prognosis (16). For instance, compared with normal breast tissues, the expression levels of Per1 and Per2 genes may decrease in sporadic and familiar breast tumours (17). When compared with sporadic forms, the Perl gene has lower expression levels, and its form is similar to that of breast cancer. This means that a potential deregulation of the circadian clock may result in the inherited form of the disease (16). The breast cancer cells may survive due to the methylation of Per1 and Cryl gene promoters, inactivating expression of these genes and disrupting the circadian cell rhythm (18). Furthermore, factors including patient age, tumour histological grade, invasion depth, lymph node metastasis and TNM staging are also associated with Per2 protein expression in patients with breast cancer (19). Although the clinical significance and certain roles of Per2 in the malignant biological behaviour of NSCLC have been reported, including chemoresistance and radioresistance, researchers have not explored the influence of Per2 on NSCLC metastasis in vitro and in vivo (20). In the current study, the roles of Per2 were explored in vitro and a metastasis model was also established in vivo by tail vein injection. It was identified that overexpression of Per2 inhibited metastasis of NSCLC. Consistent with previous studies, the current results indicated that Per2 expression was markedly downregulated in $\sim 83.87 \%$ (26 of 31) of the NSCLC samples compared with their adjacent control tissues. Furthermore, it was identified that decreased tumour differentiation and increased lymph node metastasis were associated with decreased Per2 expression, indicating that NSCLC is closely associated with the circadian gene. In addition, these results suggest Per 2 deletion or mutation may result in tumour progression and metastasis.

Numerous studies have reported that Per2 plays an important role in the generation and maintenance of circadian rhythms. It controls $2-10 \%$ of all mammalian genes (21). For rodents, $\sim 7 \%$ of clock-controlled genes regulate cell proliferation or apoptosis $(22,23)$. In addition, it has been reported that circadian rhythms change with human and other mammalian tumour formation $(24,25)$. Furthermore, studies have suggested that decreased Per2 may disrupt normal circadian rhythms of behaviour and physiology, and could significantly increase the chance of tumour and proliferative phenotypes (26-28). These findings demonstrate that Per2 is of great importance for carcinogenesis. In the current study, Per2 expression was increased in A549 cells by transducing them with a Per2-overexpressing lentivirus and then the role of Per2 in cell proliferation, migration and invasion capacity was evaluated. It was identified that enhanced Per2 expression strongly suppressed A549 cell growth in the form of blocking the cell cycle in G1 phase and restricting cell migration and invasion. The study of NSCLC xenografts in nude mice also confirmed that tumour growth rates and volumes were markedly reduced in Per2 lentivirus-transduced A549 NSCLC cells compared with the PBS control group. These findings are consistent with previous studies and suggest that Per2 may act as an anti-oncogene in tumour progression.

The antitumour molecular mechanism of Per2 in NSCLC remains unclear. A previous study reported that the antitumour effect of the clock gene Per2 is associated with DNA damage (29). It is now well established that DNA damage 

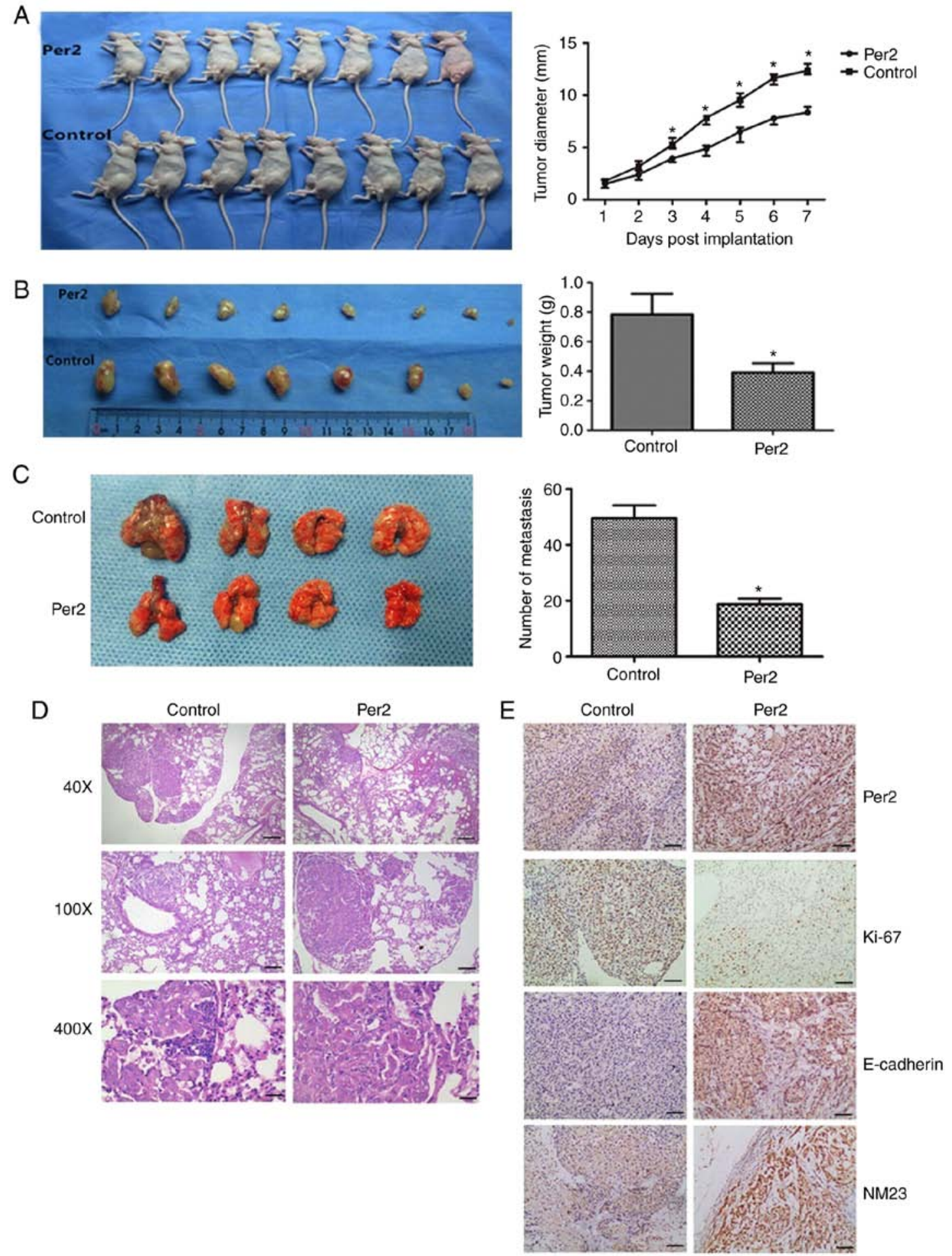

Figure 5. Overexpression of Per2 reduces tumour formation of A549 cells in a nude mouse xenograft model. The effect of Per2 on tumourigenicity in the nude mouse xenograft model was analysed in terms of (A) tumour volume and (B) tumour weight. (C and D) Lung histology in the lung metastasis mouse animal model was analysed by hematoxylin and eosin staining (magnification, $\mathrm{x} 40$ ). (E) Immunohistochemistry was performed to analyse the expression of Per2, Ki-67, E-cadherin and NM23 in mouse tumour samples (magnification, $\mathrm{x} 40$ ). ${ }^{*} \mathrm{P}<0.05$ vs. control. Per2, Period2.

depends on the activation of p53 and p53-associated transcriptional activation, including p21 (30-32). Furthermore, hypoxia of tumour cells usually results in VEGF agonist activity, which may be restrained by Per2; therefore, tumour angiogenesis may be inhibited (33). It was investigated in the current study whether the antitumour mechanism of Per2 in NSCLC was associated the p53-p21-induced DNA damage pathway.
In vitro and in vivo observations indicated that increased Per2 expression in A549 cells not only significantly increased expression of the tumour anti-oncogenes Bax, P53, and P21, but also inhibited expression of the pro-oncogenes VEGF, CD44 and c-Myc (34). These results indicate that loss of Per2 inhibits tumour cell growth and metastasis by activating the P53/P21 DNA damage-induced apoptosis pathway in NSCLC 
cells. Therefore, it was speculated that reducing Per2 may promote the proliferation and metastasis of NSCLC. This area will be investigated further in future studies.

The Per family may function as a cancer suppressor in the pathogenesis of NSCLC. However, the detailed mechanisms of the downregulation of Per still require elucidation. CpG methylation of promoter sequences may inactivate promoter functions, particularly in tumour suppressor or related genes, and may also lead to silencing of these genes. A key factor in silencing cancer suppressor genes is commonly considered to be methylation (32). Therefore, it is speculated that methylation of the promoters of Per genes may result in the inhibition of Per. Chen et al (35) noted that the abnormal expression of Period in breast cancer is not caused by genetic mutations but by the methylation of the Per1 or Per2 promoter. However, Nagel et al (36) identified that the microRNA-192/194 cluster is an effective inhibitor of the entire Per gene family using a forward genetic screen. This introduced a new mechanism to control the downregulation of Per at the post-transcriptional level. Based on the aforementioned reported, the reason for the inhibition of Per genes in cancer is complex, and not solely due to methylation. There were also certain limitations to the current study; only A549 cells were available in our laboratory at that time, therefore Per2 overexpression in A549 was studied according to the laboratory conditions. Further investigation is required to clarify the effect of Per on NSCLC development and its underlying mechanisms. In the future, further experimental studies of Per2 in NSCLC will be performed, and several cell lines will be selected to conduct a comprehensive study.

In conclusion, the present study identified that loss of Per2 may be closely associated with the development of NSCLC. Recovery of Per2 expression could suppress A549 cell growth and migration in vitro and in vivo by increasing apoptosis-associated gene expression and decreasing metastasis-associated gene expression. These findings suggest that Per2 may serve as a novel molecular target for NSCLC.

\section{Acknowledgements}

Not applicable.

\section{Funding}

The present study was supported by grants from the Scientific Research Foundation of the Technology Department of Sichuan Province, China (grant nos. 2017SZ0013 and 2018SZ0153).

\section{Availability of data and materials}

The datasets used and/or analyzed during the current study are available from the corresponding author on reasonable request.

\section{Authors' contributions}

RX and YC performed the experiments; JL, TX and YW provided technical support, critical comments and suggestions. RX, TX and XY edited the paper; RX, YC and ZW analyzed the data; XY, JL and QL designed the experiments, analyzed the data and wrote the paper. All authors read and approved the final manuscript.

\section{Ethics approval and consent to participate}

All patients provided written informed consent, and the study was approved by the Ethical Committee of Sichuan Cancer Hospital \& Institute (Sichuan, China).

\section{Patient consent for publication}

Not applicable.

\section{Competing interests}

The authors declare that they have no competing interests.

\section{References}

1. Torre LA, Siegel RL and Jemal A: Lung Cancer Statistics. Adv Exp Med Biol 893: 1-19, 2016.

2. Schabath MB, Giuliano AR, Thompson ZJ, Amankwah EK, Gray JE, Fenstermacher DA, Jonathan KA, Beg AA and Haura EB: TNFRSF10B polymorphisms and haplotypes associated with increased risk of death in non-small cell lung cancer. Carcinogenesis 34: 2525-2530, 2013

3. Siegel R, Naishadham D and Jemal A: Cancer statistics, 2013. CA Cancer J Clin 63: 11-30, 2013.

4. Ferlay J, Shin HR, Bray F, Forman D, Mathers C and Parkin DM (eds): GLOBOCAN 2008: Cancer Incidence and Mortality Worldwide. IARC Cancerbase No, 10. International Agency for Research on Cancer, Lyon, 2010.

5. Bechet D: Magnesium in health \& disease. Magnesium Res 29: 60,2016

6. Cardinali DP: The human body circadian: How the biologic clock influences sleep and emotion. Neuro Endocrinol Lett 21: 9-15, 2000.

7. Liu B, Xu K, Jiang Y and Li X: Aberrant expression of Per1, Per2 and Per3 and their prognostic relevance in non-small cell lung cancer. Int J Clin Exp Pathol 7: 7863-7871, 2014.

8. Chen-Goodspeed M and Lee CC: Tumor suppression and circadian function. J Biol Rhythms 22: 291-298, 2007.

9. Gery S, Komatsu N, Kawamata N, Miller CW, Desmond J, Virk RK, Marchevsky A, Mckenna R, Taguchi H and Koeffler HP: Epigenetic silencing of the candidate tumor suppressor gene Perl in non-small cell lung cancer. Clin Cancer Res 13: 1399-1404, 2007.

10. Couto P, Miranda D, Vieira R, Vilhena A, De Marco L and Bastos-Rodrigues L: Association between CLOCK, PER 3 and CCRN4L with non-small cell lung cancer in Brazilian patients. Mol Med Rep 10: 435-440, 2014.

11. Livak KJ and Schmittgen TD: Analysis of relative gene expression data using real-time quantitative PCR and the $2^{-\Delta \Delta C_{\mathrm{T}}}$ method. Methods 25: 402-408, 2001.

12. Retnam L, Chatikavanij P, Kunjara P, Paramastri YA, Goh YM, Hussein FN, Mutalib AR and Poosala S: Laws, Regulations, guidelines and standards for animal care and use for scientific purposes in the countries of Singapore, Thailand, Indonesia, Malaysia, and India. ILAR J 57: 312-323, 2016.

13. Chung S, Son GH and Kim K: Circadian rhythm of adrenal glucocorticoid: Its regulation and clinical implications. Biochim Biophys Acta 1812: 581-591, 2011.

14. Savvidis $C$ and Koutsilieris M: Circadian rhythm disruption in cancer biology. Mol Med 18: 1249-1660, 2012.

15. Winter SL, Bosnoyan-Collins L, Pinnaduwage D and Andrulis IL: Expression of the circadian clock genes Perl and Per2 in sporadic and familial breast tumors. Neoplasia 9: 797-800, 2007.

16. Kuo SJ, Chen ST, Yeh KT, Hou MF, Chang YS, Hsu NC and Chang JG: Disturbance of circadian gene expression in breast cancer. Virchows Archiv 454: 467-474, 2009.

17. Chu LW, Zhu Y, Yu K, Zheng T, Yu H, Zhang Y, Sesterhenn I, Chokkalingam AP, Danforth KN, Shen MC, et al: Variants in circadian genes and prostate cancer risk: A population-based study in China. Prostate Cancer Prostatic Dis 11: 342-348, 2008.

18. Hwang-Verslues WW, Chang PH, Jeng YM, Kuo WH, Chiang PH, Chang YC, Hsieh TH, Su FY, Lin LC, Abbondante S, et al: Loss of corepressor PER2 under hypoxia up-regulates OCT1-mediated EMT gene expression and enhances tumor malignancy. Proc Natl Acad Sci USA 110: 12331-12336, 2013. 
19. Miller BH, McDearmon EL, Panda S, Hayes KR, Zhang J, Andrews JL, Antoch MP, Walker JR, Esser KA, Hogenesch JB and Takahashi JS: Circadian and CLOCK-controlled regulation of the mouse transcriptome and cell proliferation. Proc Natl Acad Sci USA 104: 3342-3347, 2007.

20. Chi C, He ZF, Liu Y, Lin XM and Sun CC: Expression and clinical significance of circadian gene Per2 in non-small cell lung cancer. Zhonghua Zhong Liu Za Zhi 35: 129-131, 2013 (In Chinese).

21. McQueen CM, Schmitt EE, Sarkar TR, Elswood J, Metz RP Earnest D, Rijnkels $M$ and Porter WW: PER2 regulation of mammary gland development. Development 145: pii: dev157966, 2018.

22. Fu L and Lee CC: The circadian clock: Pacemaker and tumour suppressor. Nat Rev Cancer 3: 350-361, 2003

23. Takahashi JS, Hong HK, Ko CH and McDearmon EL: The genetics of mammalian circadian order and disorder: Implications for physiology and disease. Nat Rev Genet 9: 764-775, 2008.

24. Levi F, Okyar A, Dulong S, Innominato PF and Clairambault J Circadian timing in cancer treatments. Annu Rev Pharmacol Toxicol 50: 377-421, 2010.

25. Kelleher FC, Rao A and Maguire A: Circadian molecular clocks and cancer. Cancer Lett 342: 9-18, 2014.

26. Fu L and Kettner NM: The circadian clock in cancer development and therapy. Prog Mol Biol Transl Sci 119: 221-282, 2013.

27. Fu L, Pelicano H, Liu J, Huang P and Lee C: The circadian gene Period2 plays an important role in tumor suppression and DNA damage response in vivo. Cell 111: 41-50, 2002.

28. Younger ST, Kenzelmann-Broz D, Jung H, Attardi LD and Rinn JL: Integrative genomic analysis reveals widespread enhancer regulation by $\mathrm{p} 53$ in response to DNA damage. Nucleic Acids Res 43: 4447-4462, 2015.
29. Garner E and Raj K: Protective mechanisms of p53-p21-pRb proteins against DNA damage-induced cell death. Cell Cycle 7: 277-282, 2008.

30. Mirzayans R, Andrais B, Scott A and Murray D: New insights into p53 signaling and cancer cell response to DNA damage: Implications for cancer therapy. J Biomed Biotechnol 2012: 170325, 2012.

31. Okabe T, Kumagai M, Nakajima Y, Shirotake S, Kodaira K, Oyama M, Ueno M and Ikeda M: The impact of HIF1 $\alpha$ on the Per2 circadian rhythm in renal cancer cell lines. PLoS One 9: e109693, 2014.

32. Miyazaki K, Wakabayashi M, Hara Y and Ishida N: Tumor growth suppression in vivo by overexpression of the circadian component, PER2. Genes Cells 15: 351-358, 2010.

33. Clark SJ and Melki J: DNA methylation and gene silencing in cancer: Which is the guilty party? Oncogene 21: 5380-5387, 2002.

34. Gotoh T, Vila-Caballer M, Santos CS, Liu J, Yang J and Finkielstein CV: The circadian factor Period 2 modulates p53 stability and transcriptional activity in unstressed cells. Mol Biol Cell 25: 3081-3093, 2014.

35. Chen ST, Choo KB, Hou MF, Yeh KT, Kuo SJ and Chang JG: Deregulated expression of the PER1, PER2 and PER3 genes in breast cancers. Carcinogenesis 26: 1241-1246, 2005.

36. Nagel R, Clijsters L and Agami R: The miRNA-192/194 cluster regulates the Period gene family and the circadian clock. FEBS J 276: 5447-5455, 2009. 